\title{
BIG Data Analytics: A boon for SMART Healthcare
}

\section{Opinion}

Volume 2 Issue 1- 2021

\author{
Author Details \\ R Biswas* \\ Department of Physics, Tezpur University, Tezpur, India \\ *Corresponding author \\ R Biswas, Department of Physics, Tezpur University, Tezpur, India
}

Article History

Received: February 06, 2021 Accepted: February 10, 2021 Published: February 11, 2021

\begin{abstract}
This concise communication deals with BIG data analytics. It gives a brief outline how the health sector can be benefited from the effective use of it. Citing a recent application in combating Covid-19, we assert that BIG data can go a long way in the domain of SMART healthcare.
\end{abstract}

\section{Opinion}

BIG data of late has become a recent trend that shows a steady growth in tandem with development in information technology. Referring to voluminous information, this can be game changer in the healthcare. In this sector, BIG data engages the enormous data spanning biological, clinical, and environmental as well as lifestyle information concerning large individuals, which corresponds to their health and wellbeing along a time span. As such, emphasis is laid on the parameters involving size and volume. Revolving around three technical terms, namely, variety, veracity and velocity, BIG data handles a mammoth information. To further bolster the activity of Big Data, Internet of Things (IoT) goes in synchrony with it.

Of late, IoT has become a common technological term for big enterprises. With the advent of smart objects growing at a rate higher than the population of world, IoT has now become an obligatory part of the modern era. However, it goes with a caution of acceptability followed by adaptability [1-9]. In short, Big Data Analytics refers to effective integration and efficient analysis of various forms of data over a period; thus, catering to some impending problems. One very recent application of BIG data is towards finding effective solution for contending the ongoing pandemic.

Since the outbreak of Covid-19 in Wuhan, China, the pandemic as declared by WHO has totally jolted the global scenario. Last year, the world had come to a halt because of the continued lockdown. Not only developing nations but also the developed nations has come under the devastating grip of this. The SARS-COV-2 virus has caused a sizeable fatality worldwide and still, there is still the counter going on; albeit in a slower rate. All big pharma as well as drug companies are in a dilemma in the journey of finding best vaccine for this [8-11]. Although couple of organizations have somehow been able to come up with vaccination, the side effects as well as growing confusion of administering vaccine to various age groups cannot be ruled out. In this context, IoT driven BIG data analytics has been widely used by health professionals to find out best remedies in the fight against Covid-19 [12-19].

In one of the remarkable use of BIG data analytics, we can cite the massive use of Supercomputer Taking part in data analysis part for Covid-19. We can cite the well-known IBM’ Blue Gene supercomputer. It has been endowed with a supreme computing facility. As per reports, it efficiently surpassed the petascale barrier around sixteen years ago. Accordingly, this supercomputer played a crucial role in analyzing the sequencing of human genome, thereby paving way for designing novel drugs and treatments. Clinicians were quite bamboozled by the rapid spread of Covid-19; infecting millions of people globally. To track down it, the Dept of Energy of United States of America, which has been severely affected by this Covid-19, deployed a powerful ally-the IBM-built Summit supercomputer in combating COVID-19 [19]. In general, the infection of cells by virus is caused by an injection of spike to genetic material belonging to the host cells. The practitioners in wet labs patiently inspect the reaction of the microorganism in response to new compounds. Although, this kind of practices although bears fruit; in terms of time and handling, they may prove cumbersome. In such cases, computer simulations will not only save ample amount of time but also provide unique solutions. In the cautionary aspect, these simulations being endowed with the ability of analyzing reactions of different virus corresponding to several variables handle terabytes of data pertaining to each variable. Consequently, the multiple simulations emerge out to be a very tedious process in the context of time as well as hardware involved. Accordingly, through an efficient IoT enabled Big data analytics scheme, Summit helped researchers to simulate approximately 8,000 compounds in a short span of time. The main goal was to identify the optimum model for constraining the infections of SARSCOV-2. As such, seventy-seven small-molecule compounds, such as medications and natural compounds have been identified; exhibiting 
ability of weakening COVID-19's ability to dock with and infect host cells. Although, these finding are not a direct mean to cure the viral disease; however, the results will be a direct boon for future studies and provide a basis such that experimentalists will deploy these compounds and find the best suitable one as a potential tool for mitigating the COVID-19[19]. This is just one of the very recent uses of BIG data analytics. In many occasion, BIG data analytics has been found to be useful in prescribing drugs as well as medications.

Conclusively, BIG data analytics can play a vital role in SMART healthcare. However, we cannot utilize its full potential if we are not well aware of targeted research endeavor. Security and quality of big data arise as major challenges. When there occurs synergy of privacy and ethical principles in monitoring BIG data, use can have a deeper insight in understanding the objectives of implementation and quality, which will eventually, lead to developed and optimized care processes, early diagnosis etc. leading to SMART healthcare.

\section{References}

1. Akgül, Ceyhun Burak (2011) Content-based image retrieval in radiology: current status and future directions. Journal of Digital Imaging 24(2): 208-222.

2. Bertsimas D, Bjarnadóttir M, Kryder J, Pandey R, Vempala S, et al., (2008) Algorithmic prediction of health-care costs. Operations Research 56(6): 1382-1557.

3. Bhattacherjee A, Hikmet N (2007) Physicians' resistance toward healthcare information technology: a theoretical model and empirical test. European Journal of Information Systems: Including a Special Section on Healthcare Information 16: 725-737.

4. LeRouge C, Mantzana V, Vance Wilson E (2007) Healthcare information systems research, revelations and visions. European Journal of Information Systems 16: 669- 671.

5. Müller Henning (2004) A review of content-based image retrieval systems in medical applications-clinical benefits and future directions. International journal of medical informatics 73(1): 1-24.

6. Shmueli G, Koppius O (2011) Predictive analytics in information systems research. MIS Quarterly, 35(3): 553-572.

7. Sarkar, Indra Neil (2010) Biomedical informatics and translational medicine. Journal of Translational Medicine 8(1): 22.
8. Walker M, Fonda SJ, Salkind S, Vigersky R (2012) Advantages and disadvantages of real-time continues glucose monitoring in people with type 2 diabetes. Blood glucose monitoring 8(1): 25 .

9. Hao Wang, Ottar L Osen, Guoyuan Lit, Wei Lit, Hong-Ning Dai, et al., (2015) Big Data and Industrial Internet of Things for the Maritime Industry in Northwestern Norway.

10. Zheng Li, Diego Seco, Alexis Eloy Sánchez Rodríguez (2019) Microservice-Oriented Platform for Internet of Big Data Analytics: A Proof of Concept, Sensors 9(19): 1134.

11. Darabseh A, Al-Ayyoub M, Jararweh Y, Benkhelifa E, Vouk M, et al., (2015) Storage: A Software Defined Storage Experimental Framework. In Proceedings of the 3rd International Conference on Cloud Engineering (IC2E 2015),

12. Tempe AZ (2015) Computer Society: Tempe, AZ, USA, 13: 341-346.

13. Djedouboum AC, Ari AAA, Gueroui AM, Mohamadou A, Aliouat Z (2018) Big Data Collection in Large-Scale Wireless Sensor Networks. Sensors 18: 4474.

14. Kang JM, Bannazadeh H, Rahimi H, Lin T, Faraji M, et al., (2013) Software-Defined Infrastructure and the Future Central Office. In Proceedings of the $2^{\text {nd }}$ Workshop on Clouds Networks and Data Centers, Budapest, Hungary 9-13: 225-229.

15. Kang JM, Lin T, Bannazadeh H, Leon-Garcia A (2014) Software-Defined Infrastructure and the SAVI Testbed. In Trident Com 2014: Testbeds and Research Infrastructure: Development of Networks and Communities; Leung VC, Chen M, Wan J, Zhang Y (Eds.) Springer: Cham, Switzerland 137: 3-13.

16. Li CS, Brech BL, Crowder S, Dias DM, Franke H, et al., (2014) Software defined environments: An introduction. IBM J Res De, 58: 1-11.

17. Nunes BAA, Mendonca M, Nguyen XN, Obraczka K, Turletti T (2014) A Survey of Software-Defined Networking: Past, Present, and Future of Programmable Networks, IEEE Commun Surv Tutor, 16: 1617-1634.

18. Villari M, Fazio M, Dustdar S, Rana O, Ranjan R (2016) Osmotic Computing: A New Paradigm for Edge/Cloud Integration, IEEE Cloud Comput 3: 76-83.

19. Vögler M, Schleicher JM, Inzinger C, Dustdar S Ahab (2017) A Cloudbased Distributed Big Data Analytics Framework for the Internet of Things, Softw Pract Exp 47: 443-454. 